\title{
Viewpoint: The Current Advances and Future Optimization of Blood-based Tumor Mutation Burden as a Biomarker for Cancer Immunotherapy
}

Anlin $\mathrm{Li}^{1,2}$

${ }^{1}$ The First Clinical Medical College, Guangdong Medical University, Zhanjiang, Guangdong, China. ${ }^{2}$ Department of Oncology, Sun Yat-sen Memorial Hospital, Sun Yat-sen University, Guangzhou, Guangdong, China

Correspondence to: Anlin Li, The First Clinical Medical College, Guangdong Medical University, No. 2 Wenming East Road, Xiashan District, Zhanjiang 524023, People's Republic of China. E-mail: anlinli.napert@gmail.com; ORCID: https://orcid.org/0000-0002-2717-7768

Keywords: blood-based mutation burden (bTMB); cancer immunotherapy; immune checkpoint inhibitor (ICI); biomarker; mutation

\begin{abstract}
Instead of tissue-based detection, blood-based tumor mutation burden (bTMB) is becoming an alternative promising alternate to predict the response of immune checkpoint inhibitor in cancers, especially non-small-cell lung cancer. Although bTMB is more convenient and less invasive, many evidences identified its limited predictive ability and less accurate discrimination of candidates to receive immunotherapy. Several ways of adjustments have been applied to improve the clinical usefulness of bTMB, such as setting restriction for threshold of allele frequency to exclude some unwanted mutations. But many questions remained to be explored such as the number and the type of mutations that should be incorporated into the bTMB estimation. This viewpoint summarized the current attempts to modify bTMB and provided granular aspects that have implications for further enhancement of bTMB's predictive capability.
\end{abstract}




\section{Introduction}

The blood-based tumor mutation burden (bTMB) has emerged as a novel approach to predict the efficacy of immune checkpoint inhibitor (ICI) for non-small-cell lung cancer (NSCLC) [1,2]. The advantages of bTMB over tissue-based TMB (tTMB) include less invasive damage, more obtainable convenience, ability to dynamic multiple testing during treatment, and more comprehensive reflection of genetic heterogeneity in the tumor microenvironment of both primary and metastatic sites. This viewpoint illustrates the current advances to improve the predictive utility of bTMB and deeper aspects that merit consideration in future optimization of bTMB-based biomarker.

\section{Current Advances}

The article by Wang et al. [3] showed that the original bTMB score [1] failed to discriminate overall survival (OS) benefits of PD-L1 inhibitor atezolizumab among patients with NSCLC. Their team speculated that circulating tumor DNA (ctDNA) maximum somatic allele frequency (MSAF) as a negative prognostic factor for OS might interfere with the positive predictive effect of bTMB, and found mutations with high AF were associated with increased MSAF, responsible for weaker predictive ability of bTMB. Therefore, they constructed a more effective bTMB algorithm by setting an AF threshold of less than 5\% (LAF-bTMB score), which represented lower correlation with MSAF and was significantly associated with OS benefit in ICI versus chemotherapy and in the ICI group. More importantly, this algorithm expanded the population that might benefit from ICI over chemotherapy; 181 out of 853 (21.2\%) patients who were previously classified as low bTMB by the original score were reclassified into high LAF-bTMB group and could be recommended to receive ICI.

Moreover, Liu et al. considered that mutations with minor AF, identified by low AF/MASF value, were likely minor subclones, which might also negatively affect bTMB prediction [4]. Thus, they further redefined the bTMB algorithm by concurrently excluding both high and minor AF values [5]. The mutations with $\mathrm{AF} \leq 5 \%$ and $\mathrm{AF} / \mathrm{MASF} \geq 10 \%$ were included for establishment of modified $\mathrm{bTMB}$. Although the difference in survival between ICI and chemotherapy is more pronounced by applying this modified bTMB compared with applying the LAF-bTMB score, the beneficiaries were narrowed down by 41 patients.

The MSAF is the highest AF among all detected somatic variants, commonly used as a surrogate to estimate ctDNA amount and tumor content. Patients with high MSAF were shown to have great 
tumor heterogeneity and advanced tumor stage, which were associated with inferior prognosis and immunotherapy efficacy [6,7]. Yu et al. conducted individual patient data meta-analysis evaluating the bTMB and MSAF in predicting survival of ICI versus docetaxel among EGFR wild-type NSCLC patients [8]. Using a previously-established bTMB cut-point 16 [1], they observed paradoxical results that patients with a $\mathrm{bTMB}<16$ significantly prolonged OS but not progression-free survival (PFS), whereas patients with a $b T M B \geq 16$ had evident enhancement in PFS but not OS. However, they found MSAF could stratify patients perfectly, suggesting the crucial role of MSAF in determining immunotherapeutic effect. Hence, Yu et al. tried to build a more comprehensive blood-based mutational signature by combining bTMB and MSAF. The bTMB-MSAF score markedly improved the degree of correlation with OS compared with bTMB alone $\left(R^{2}=0.90\right.$ versus 0.68$)$, meaning that $22 \%$ predictive value was additionally provided by adding MSAF to bTMB [8].

A high MSAF is the prerequisite to precise detection of variants and a low MSAF has been shown to greatly contribute to the discordance between bTMB and tTMB [1,9]. Chen et al. surprisingly found that NSCLC patients with both low bTMB and low MSAF could derive survival benefits from ICI over chemotherapy [10], in accordance with another study showing both low and high bTMB were associated with better OS than medium bTMB [11]. These results suggested that a proportion of patients might be wrongly classified into low-bTMB group due to a low MSAF value.

\section{Remaining Questions and Future Optimization}

3.1. What is the optimal gene panel size for bTMB calculation? Using the targeted panel-based nextgeneration sequencing to estimate bTMB is more cost-effective than using the whole exon sequencing (WES). However, the risk of imprecise evaluation of mutation level should not be neglected. Budczies et al. recommended the panel size should be at least $1 \mathrm{Mb}$ to better avoid classification of patients [12]. Current studies investigating the predictive value of bTMB utilized various panel sizes $[1,2,7,13]$. While the panel to estimate tTMB has been relatively well-established, the optimal gene panel size for bTMB calculation is far from standardization [13].

3.2. Which mutation type should be included and should they be equally considered? The mutation types used for calculation of bTMB varied among different studies [13]. The prior research [1] included only single nucleotide variants (SNVs) without considering insertions and deletions (indels), so a 
patient's bTMB might be underestimated and if he harbors a large number of indels, which might result in suboptimal treatment decision. In addition, the extent of immune response elicited by anti-PD-1 therapy was shown to be particularly dependent on indel load [14], highlighting the need to include these mutations into bTMB estimation. Additionally, most bTMB calculation was based only on nonsynonymous mutations [13], but a study by Wang et al. [2] additionally included synonymous mutations and surprisingly observed a higher correlation between bTMB and WES-based TMB. The biological heterogeneity among different mutation types should be further clarified.

\subsection{How to adjust the inconsistent predictive effects among mutated genes and alleles? A recent study} revealed a non-linear association of bTMB with OS benefits [11]. A potential explanation is that different mutations might produce neoantigens with diverse levels of immunogenicity and most neoantigens do not possess higher immunogenicity than the wild-type [15]. Only few immunodominant neoantigens contribute to the immunotherapy benefits, while majority of neoantigens tend to evade immune surveillance. It's conceivable that some low-immunogenicity mutations could result in increased bTMB but fail to generate potent immune response and therefore offset the positive predictive effects of immunogenic mutations. For instance, TP53 mutation was associated high CD8 $+\mathrm{T}$ cell infiltration and longer PFS of patients treated with immunotherapy, but an addition co-occurring STK11 mutation greatly reduced CD8+ T cell density and reversed the predictive impact of TP53 mutation [16]. However, some published panels such as MSK-IMPACT and FoundationOne CDx still equally incorporated STK11 into bTMB calculation [17,18]. In addition to gene-level differences, the effect of a mutation could vary by allele; for instance, patients with different EGFR mutation subtypes respond distinctly to ICI $[19,20]$. Therefore, adjusting levels of immunogenicity for different mutations and alleles could enhance the predictive utility of bTMB, probably through assigning those non-immunogenic mutations a negative number.

3.4. How could bTMB threshold and gene panel design for particular situation? The mutations that could produce ICI-targeted immunodominant neoantigens probably vary among different clinical conditions with respect to cancer types and subtypes, immunotherapy type, treatment setting, sex, and histology, etc. Therefore, the optimal gene panel and bTMB threshold should be better adjusted for each situation. A first-line B-F1RST trial [21] tried to predict survival with atezolizumab by using 
bTMB cut-point 16, which was identified in the prior study based on subsequent-line patients [1], and they failed to obtain significant results for OS or PFS. Dynamic change of ctDNA load and bTMB from treatment-naïve patients to previously-treated patients might account for this inconsistency. Hence, caution should be taken when applying bTMB threshold in distinct treatments or populations. Extending the clinical utility of bTMB from current practice in NSCLC to pan-cancer requires cancerspecific panels to be designed.

3.5. Could selected mutated genes used to be a surrogate biomarker to bTMB? Yu et al. [8] identified ATM, KEAP1, TP53 as significant predictor of ICI efficacy and combined these mutation statuses with clinicopathological information to construct a clinicopathologic-genomic nomogram. Notably, the nomogram exhibited better clinical usefulness than bTMB in decision curve analysis. Previous researches have successfully used combination of two to three mutations as dichotomous biomarker to display distinct tumor microenvironment patterns and predict ICI efficacy [16,22], although these mutations were derived from tissue. Screening of blood-based co-mutations will be a more simple and convenient approach to classify candidates for immunotherapy.

\section{Conclusions}

Current modifications of bTMB are not enough and the aforementioned heterogeneous characteristics should be considered to develop an optimal bTMB predictive tool to realize more accurate selection of candidates to receive immunotherapy. 
Author Contributions: Anlin Li contributes to the whole content.

Conflicts of Interest: The author declares no conflict of interest.

Acknowledgments: This work was supported by National Students' Innovation and Entrepreneurship training program (grant number 201910571001); Special Funds for the Cultivation of Guangdong College Students' Scientific and Technological Innovation (grant number pdjh2019a0212); and Guangdong Medical University College Students' Innovation Experiment Project (grant number ZZZF001). 


\section{References}

1. Gandara DR, Paul SM, Kowanetz M, et al. Blood-based tumor mutational burden as a predictor of clinical benefit in non-small-cell lung cancer patients treated with atezolizumab. Nat Med. 2018;24(9):1441-1448. doi:10.1038/s41591-018- 0134-3

2. Wang Z, Duan J, Cai S, et al. Assessment of blood tumor mutational burden as a potential biomarker for immunotherapy in patients with non-small cell lung cancer with use of a next-generation sequencing cancer gene panel. JAMA Oncol. 2019;5(5):696-702. doi:10.1001/jamaoncol.2018.7098

3. Wang Z, Duan J, Wang G, et al. Allele frequency-adjusted blood-based tumor mutational burden as a predictor of overall survival for non-small cell lung cancer patients treated with PD-1/PD-L1 inhibitors. J Thorac Oncol. 2020;15(4):556-567

4. Liu Z, Xie Z, Zhao S, et al. Presence of allele frequency heterogeneity defined by ctDNA profiling predicts unfavorable overall survival of NSCLC. Transl Lung Cancer Res 2019;8(6):1045-1050

5. Liu Z, Xie Z, Cai X, et al. A Modified Algorithm Adjusting Both High and Minor Allele Frequency Mutation to Redefine Blood-Based Tumor Mutational Burden (bTMB) for Optimal Prediction of Clinical Benefits From Immune Checkpoint Inhibitor Therapy. J Thorac Oncol. 2020;15(5):e69-e72

6. Schwaederle M, Husain H, Fanta PT, et al. Use of Liquid Biopsies in Clinical Oncology: Pilot Experience in 168 Patients. Clin Cancer Res. 2016;22(22):5497-5505. doi:10.1158/1078-0432.CCR16-0318

7. Chae YK, Davis AA, Agte S, et al. Clinical Implications of Circulating Tumor DNA Tumor Mutational Burden (ctDNA TMB) in non-small cell lung cancer. Oncologist. 2019;24(6):820-828. doi:10.1634/theoncologist.2018-0433.

8. Yu Y, Chen Y, Li A, et al. Novel blood-based tumor mutation algorithm and nomogram predict survival of immune checkpoint inhibitor in non-small-cell lung cancer: Results from two multicenter, randomized clinical trials. Clin Transl Med. 2020;10(2):e53.

9. Zhou C, Yuan Z, Ma W, et al. Clinical utility of tumor genomic profifiling in patients with high plasma circulating tumor DNA burden or metabolically active tumors. J Hematol Oncol. (2018) 11:129. doi: 10.1186/s13045-018-0671-8

10. Chen YT, Seeruttun SR, Wu XY, Wang ZX. Maximum Somatic Allele Frequency in Combination With Blood-Based Tumor Mutational Burden to Predict the Efficacy of Atezolizumab in Advanced Non-small Cell Lung Cancer: A Pooled Analysis of the Randomized POPLAR and OAK Studies. Front Oncol. 2019;9:1432. doi:10.3389/fonc.2019.01432

11. Nie W, Qian J, Xu MD, et al. A non-linear association between blood tumor mutation burden and prognosis in NSCLC patients receiving atezolizumab. Oncoimmunology. 2020;9(1):1731072. doi:10.1080/2162402X.2020.1731072 
12. Budczies J, Allgäuer M, Litchfield K, et al. Optimizing panel-based tumor mutational burden (TMB) measurement. Ann Oncol. 2019;30(9):1496-1506. doi:10.1093/annonc/mdz205

13. Fenizia F, Pasquale R, Roma C, Bergantino F, Iannaccone A, Normanno N. Measuring tumor mutation burden in non-small cell lung cancer: tissue versus liquid biopsy. Transl Lung Cancer Res. 2018;7(6):668-677. doi:10.21037/tlcr.2018.09.23

14. Mandal R, Samstein RM, Lee KW, et al. Genetic diversity of tumors with mismatch repair deficiency influences anti-PD-1 immunotherapy response. Science. 2019;364(6439):485-491. doi:10.1126/science.aau0447

15. Castle J, Kreiter S, Diekmann J, et al. Exploiting the Mutanome for Tumor Vaccination. Cancer Res. 2012;72(5):1081-91

16. Biton J, Mansuet-Lupo A, Pecuchet N, et al. TP53, STK11, and EGFR Mutations Predict Tumor Immune Profile and the Response to Anti-PD-1 in Lung Adenocarcinoma. Clin Cancer Res. 2018;24(22):5710-5723

17. Cheng DT, Mitchell TN, Zehir A, et al. Memorial Sloan Kettering-Integrated Mutation Profiling of Actionable Cancer Targets (MSK-IMPACT): A Hybridization Capture-Based Next-Generation Sequencing Clinical Assay for Solid Tumor Molecular Oncology. J Mol Diagn. 2015;17(3):251-264. doi:10.1016/j.jmoldx.2014.12.006

18. Frampton GM, Fichtenholtz A, Otto GA, et al. Development and validation of a clinical cancer genomic profiling test based on massively parallel DNA sequencing. Nat Biotechnol. 2013;31(11):1023-1031. doi:10.1038/nbt.2696

19. Hastings $\mathrm{K}, \mathrm{Yu} \mathrm{H}$, Wei W, et al. EGFR mutation subtypes and response to immune checkpoint blockade treatment in non-small cell lung cancer. Ann Oncol. 2019;30(8):1311-1320

20. Haratani K, Hayashi H, Tanaka T, et al. Tumor immune microenvironment and nivolumab efficacy in EGFR mutation-positive non-small-cell lung cancer based on T790M status after disease progression during EGFR-TKI treatment. Ann Oncol. 2017;28(7):1532-1539. doi:10.1093/annonc/mdx183

21. M. Socinski, V. Velcheti, T. Mekhail, et al. LBA83 - Final efficacy results from B-F1RST, a prospective phase II trial evaluating blood-based tumour mutational burden (bTMB) as a predictive biomarker for atezolizumab (atezo) in 1L non-small cell lung cancer (NSCLC). ESMO. doi: 10.1093/annonc/mdz394.081.

22. Dong ZY, Zhong WZ, Zhang XC, et al. Potential predictive value of TP53 and KRAS mutation status for response to PD-1 blockade immunotherapy in lung adenocarcinoma. Clin Cancer Res. 2017;23(12):3012-3024. doi:10.1158/1078-0432.CCR-16-2554 